\section{Materials Research Society Fall Meeting offers mix of traditional events and new activities}

\author{
www.mrs.org/fall2014 \\ www.mrs.org/OnDemand ${ }^{\circledR}$
}

$\mathrm{T}^{\mathrm{n}}$ he Materials Research Society (MRS) 2014 Fall Meeting and Exhibit featured over 6000 presentations, including more than 3300 oral presentations and nearly 3000 poster presentations on November 30 through December 5 in Boston, Mass. The Meeting Chairs, Husam N. Alshareef (King Abdullah University of Science and Technology), Amit Goyal (Oak Ridge National Laboratory), Gerardo Morell (University of Puerto Rico), José A. Varela (University of São Paulo State), and In Kyeong Yoo (Samsung Advanced Institute of Technology), compiled 52 technical symposia organized into six topics: Biomaterials and Soft Matter; Electronics and Photonics; Energy and Sustainability; Nanomaterials and Synthesis; Theory, Characterization, and Modeling; and General Materials and Methods.

To complement these sessions, tutorials were offered in several technical areas, and poster sessions were held during the evenings. The international exhibit showcased products and services of interest to the materials community. Federal agencies hosted sessions on government funding policies and practices. The Meeting also featured the Science as Art competition, science presentations, a NASA Biological Materials, Biomaterials, and Biomimetics Workshop, and sustainability events. The special talks delivered by the Society's award recipients were well attended.

MRS hosted undergraduate students from NSF's Partnerships for Research and Education in Materials (PREM) Program. This year, the invited PREM students not only participated in mentoring and professional development sessions, but also celebrated the PREM 10th anniversary. The Technology Innovation Forum added a new program - iMatSciInnovation in Materials Science - that provided a demonstration platform for technology providers across all science, technology, engineering, and mathematics departments at universities and start-ups to showcase their newest materials-focused technologies.

New to the Meeting this year was the Materials Hackathon. Participants made 30-second pitches of ideas for new materials-related software, formed teams around those ideas, brought the concepts to life with working code, and presented the results to a panel of judges from across the materials field.

The Career Fair included on-site interviews, mentoring, workshops, resume critiques, mock interviews, and networking opportunities. Some of the related workshops included science writing, industrial careers, a technical poster design seminar, and an interactive scientific publishing session that explained the basics of the publishing process.

A special feature in the Tutorial $\mathrm{T}$ session on optoelectronics was a presentation by Hiroshi Amano, Nagoya University, on the fundamental physics of nitride-based optoelectronic devices. The Laureate of the 2014 Nobel Prize in Physics emphasized the need for lighting sources with greater efficiencies than what is currently available. Since the 2011 Fukushima accident, dwindling energy supplies in countries such as Japan are facing issues with increasing power consumption.
Nitride-based light-emitting diodes (LEDs), such as AlN, GaN, and InN, feature large piezoelectric polarization, which exhibit larger diffusion length and, consequently, higher quantum efficiencies. Amano described an emerging molecular beam epitaxy RF method that, using a multi-step process, is capable of producing samples capped with nitrides and devoid of oxidation.

Amano's research focuses on a pulsed growth approach that yields nanowire LEDs. He remains hopeful that this nanostructure approach will elevate these devices, which are already widely used in many applications, to an even greater implementation level.

Julia R. Greer of the California Institute of Technology, and recipient of the Kavli Early Career Award in Nanoscience, presented an energetic and comprehensive talk on "Threedimensional architected nanostructured meta-materials." Speaking to a full auditorium of several hundred researchers, she described how scaling scaffolded structural systems down to a microlattice, and then even further to a nanolattice, allows the development of extraordinarily strong mechanical systems. Despite being able to support very high compression loads- $99.9 \%$ of their composition is air - the beams are hollow, and the structure is light enough to be held up by the tip of a dandelion.

These properties cannot be described by traditional structural effects, as the structure becomes stronger only once its feature sizes are reduced to $10 \mathrm{~nm}$. Greer showed an example of a chalk-like piece of aluminum oxide being compressed. Although the structure in a microlattice form buckled (much like regular chalk) under sufficiently high compression, the nano-sized structure retained its integrity.

The trick to synthesizing these structures, Greer explained, involved the implementation of two-photon lithography, which relied on regions of constructive interference from the dualbeam system to etch specific holes in a photoresist. These are not limited to hollow structures - by electroplating copper into the etched spaces, three-dimensional 
(3D) conductive architectures were also achieved. Greer introduced a wide array of applications of these structures, ranging from Si-based anodes to 3D scaffolds for cell growth to photonic crystals.

At the start of Symposium X, MRS Immediate Past President Tia Benson Tolle presented the MRS Outstanding Young Investigator Award to Henry Snaith of the University of Oxford. Snaith commenced with a brief review of his research before 2012, none of which involved perovskites but some of which involved important principles that he later applied to solar cells based on these materials. He described dye-sensitized cells as a "platform technology" because sensitizing by coating $\mathrm{TiO}_{2}$ with perovskites drew directly from this approach.

He also drew from the use of mesoporous $\mathrm{TiO}_{2}$ structures to help make normally incompatible materials more compatible. The original $\mathrm{CaTiO}_{3}$ perovskite was discovered in 1839 , but it was not until 1959 that the defining structure of it and all perovskites was determined, and it took until 2006 for the first perovskite solar cell publication to

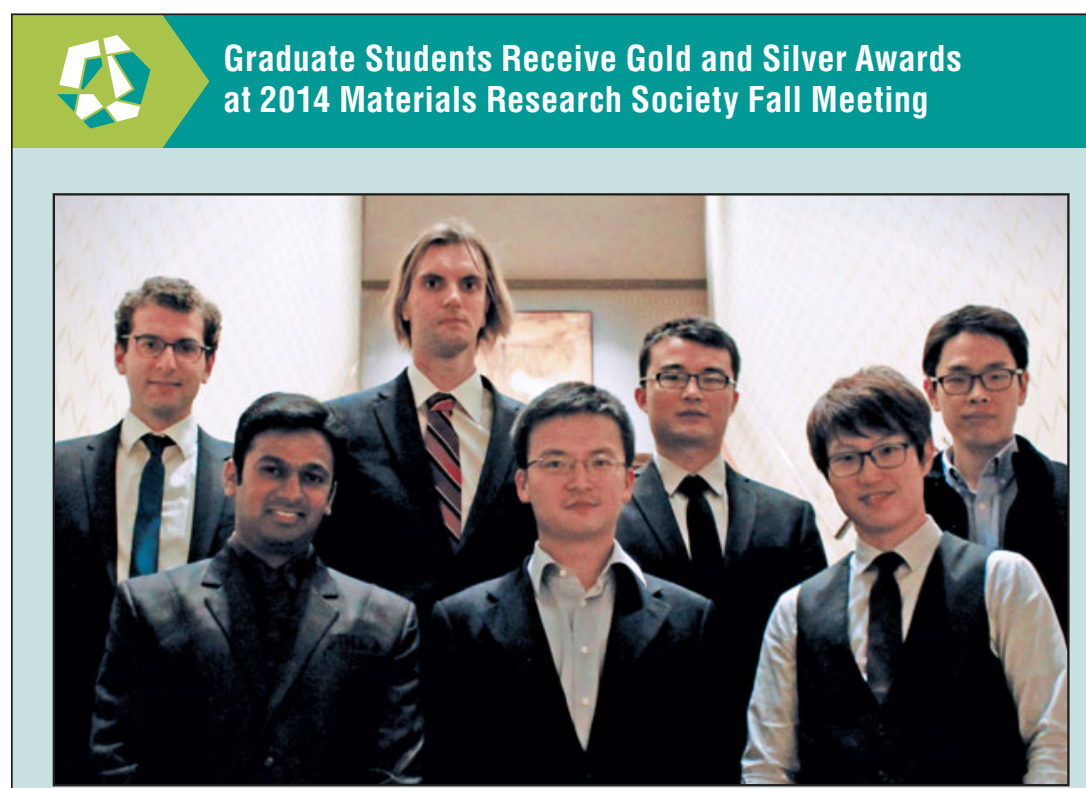

Gold Graduate Student Awards (front row, left to right): Rajesh Kappera, Huiliang Wang, and Junghoon Jahng; (second row, left to right): Max Kory, Evgeny Mikheev, Linsen Li, and Jinhyuk Lee

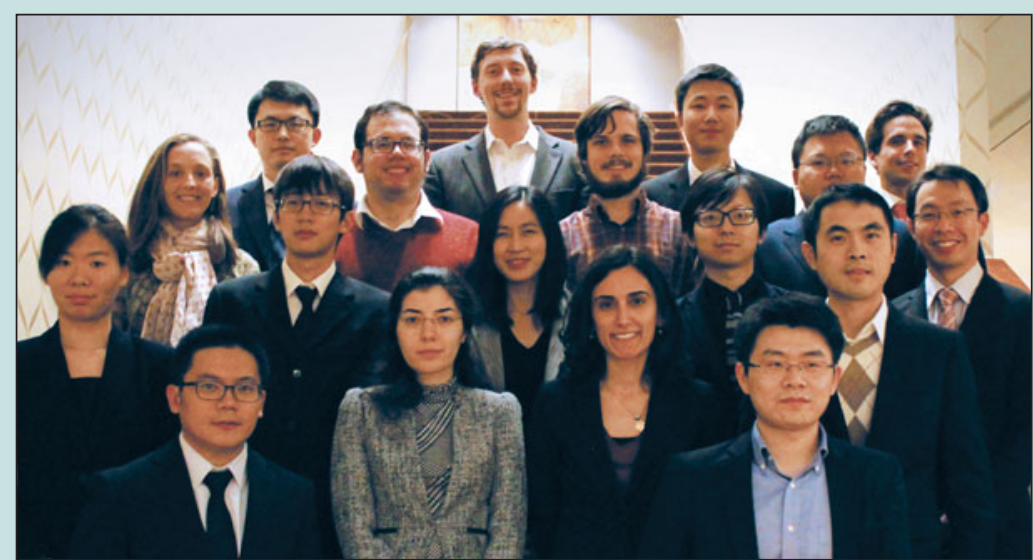

Silver Graduate Student Awards (front row, left to right): Peixuan Chen and Qiming Wang; (second row, left to right): Nan K. Li, Sara Kadkhodaei, Canan Dagdeviren, and Wesley Guangyuan Zheng; (third row, left to right): Ming Gong, Wei-Chen Wu, I-Cheng Tung, and Kwan Wee Tan; (fourth row, left to right): Kelsey Hatzell, Anthony DiLauro, Zachary Bryan, and Bo Zhang; (fifth row, left to right): Xiaonan Wen, Christopher Proctor, Junhao Lin, and Ian McCue

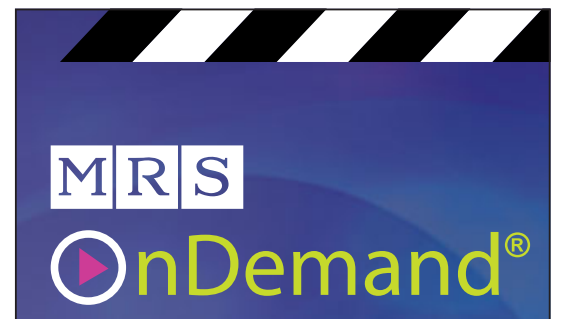

\section{New Content Now Available}

\section{THE 2014 MRS FALL MEETING ON DEMAND}

MRS OnDemand ${ }^{\circledR}$ now includes a large sampling of the rich materials science content from the 2014 MRS Fall Meeting. Watch presentations from the Meeting FREE, for a limited time, through an interactive experience, complete with video, audio and presentation materials.

Award talks by Marvin L. Cohen, Rodney S. Ruoff, Long-Qing Chen, Sharon C. Glotzer, Nicholas A. Kotov, and Mercouri G. Kanatzidis

$\checkmark$ Plenary Session

Fred Kavli Distinguished Lectureship in Nanoscience

-Kavli Early Career Award in Nanoscience Talk

- Special Roundtable Session on The Materials Genome Initiative

$>$ Symposium X-Frontiers of Materials Research

- Professional Development SessionEssentials of Getting Your Work Published

-Five Tutorial Sessions

-Select Talks from 17 Technical Symposia

\section{- "Best Poster" Nominees}

Plus, view interviews, news and highlights from the 2014 MRS Fall Meeting via MRS TV.

Whether you made it to Boston or not, now's the time to enjoy Meeting content for FREE, from your home, office or on the go. It's Your MRS, Your Way

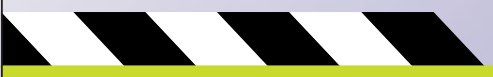

wwW.mrs.org/on-demand 
appear. Today, the emphasis is on halide perovskites, and these are what Snaith discussed in more detail.

A number of practical issues have become important as real-world applications begin to be considered. For example, perovskite films are brown, not so good for coating large buildings. Stability in sunlight, water, and high temperatures needs to be achieved. Given solutions, thin-film perovskite solar cells could become cost-competitive with silicon devices, could be less energy-intensive to produce, and would generate far less waste material, but, said Snaith, silicon technology has come so far that it is foolish to dismiss further progress.

Von Hippel Award recipient Marvin L. Cohen, from the University of California-Berkeley, began his presentation by providing a history of quantum theory as applied to solids. Given atoms, the quantum mechanical model of a nucleus with tightly bound core electrons and more loosely bound valence electrons early on was the way to explain their properties, such as the sharp lines in the optical spectra of gases. Solids with their broad spectra were a tougher case. The simple model of electrons packed in a box with a background positive potential explained the wide range of electrical resistivity values in terms of states near the bandgap or Fermi energy. Other properties awaited knowledge about states over a wider energy range, studied through optical spectra originating from interband transitions. By 1957, there was still no detailed knowledge of silicon's band structure. However, a breakthrough yielded the empirical pseudopotential method and today's ability to calculate a wide variety of properties for complex materials and nanostructures.

Cohen divided the quantum models of matter into two classes. The first was the interacting atoms model in which the "particles" making up the solid are electrons and the previously mentioned cores that interact strongly. This approach led to understanding optical properties of semiconductors from interband transitions. It also set the stage for other empirical approaches and, ultimately, ab initio methods in which the

\begin{tabular}{|c|c|}
\hline $\begin{array}{l}\text { 1-Material Inc. } \\
\text { Agilent Technologies } \\
\text { Air Force Office of Scientific Research } \\
\text { AIXTRON SE } \\
\text { Aldrich Materials Science } \\
\text { ANA Innovation Huts and Services Inc. } \\
\text { Angstrom Engineering Inc. } \\
\text { Applied Diamond, Inc. } \\
\text { Applied Materials \& Interfaces, ACS Publications } \\
\text { Applied Physics Reviews, AIP Publishing } \\
\text { Army Research Office } \\
\text { Asylum Research, an Oxford Instruments } \\
\text { Company } \\
\text { AZ Electronic Materials } \\
\text { BD Biosciences } \\
\text { Biomaterials Science \& Engineering, ACS } \\
\text { Publications } \\
\text { Biomaterials Science, RSC } \\
\text { Boston Scientific } \\
\text { Brewer Science } \\
\text { ChemAxon } \\
\text { Cline Innovations LLC } \\
\text { Council on Undergraduate Research } \\
\text { Defense Threat Reduction Agency (DTRA) } \\
\text { Department of Energy (DOE) } \\
\text { Element Six } \\
\text { Engineering and Physical Sciences Research } \\
\text { Council (EPSRC) } \\
\text { EUVL Infrastructure Development Center } \\
\text { EV Group } \\
\text { FEI Company } \\
\text { FEI Deutschland GmbH } \\
\text { Fine Abrasives Taiwan Co., LTD } \\
\text { FOM Technologies } \\
\text { Fraunhofer USA, Inc. } \\
\text { GE Global Research } \\
\text { Hamamatsu Corporation } \\
\text { Hokkaido University } \\
\text { HORIBA Scientific } \\
\text { hq graphene } \\
\text { IPen, Inc. } \\
\text { Jiangnan Graphene Research Institute }\end{array}$ & 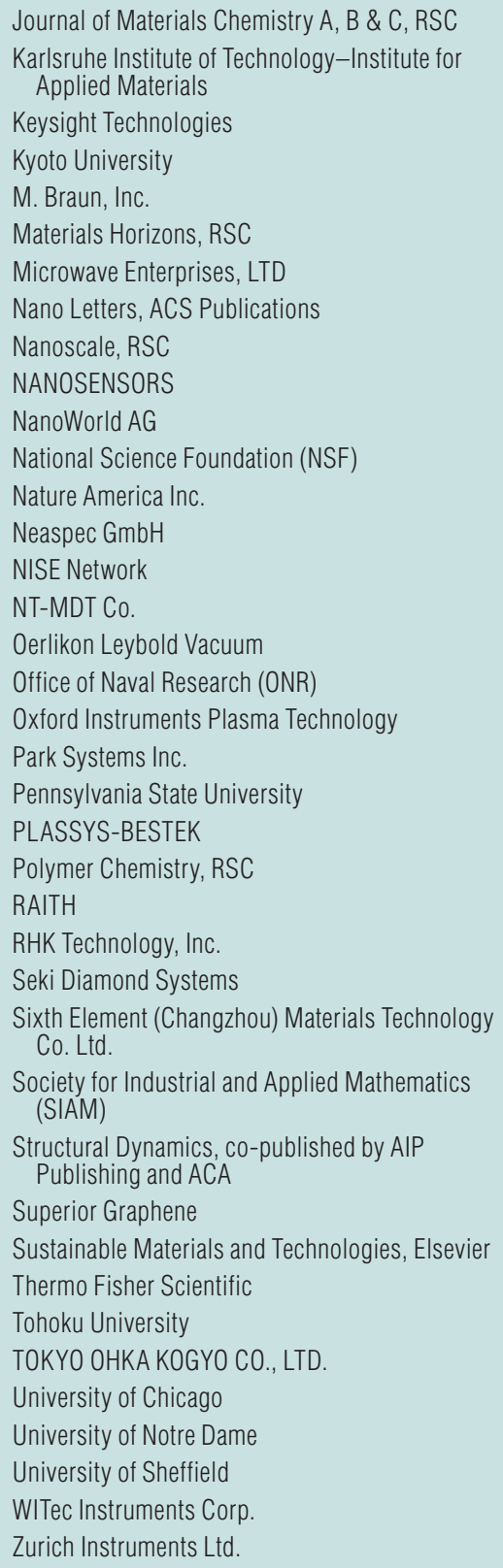 \\
\hline
\end{tabular}

only input information is that about atoms (atomic number, mass, structure).

The second way of thinking about solids, elementary excitations, was based on a probe-response concept. The excitations are "fictitious" quasiparticles that are used to explain the response to a probe. The most familiar of these are the holes left in valence bands after optical excitation. Collective excitations, such as phonons, are another example. Today's "standard" model of solids encompasses both interacting atoms and elementary interactions.

These presentations and more from the 2014 MRS Fall Meeting are available through MRS OnDemand ${ }^{\circledR}$ video capture as well as news coverage of the Meeting on MRS TV and Meeting Scene ${ }^{\circledR}$. MRS Proceedings from the Meeting are also available online. Further information can be accessed at www.mrs.org/fall2014. 